\title{
Ácaros predadores associados ao ácaro-da-erinose da lichia
}

\author{
Pedro Renan Ferreira Picoli( ${ }^{(1)}$, Marineide Rosa Vieira(1), Eloisa Aparecida da Silva ${ }^{(1)}$ \\ e Max Sander de Oliveira da Mota(1)
}

\begin{abstract}
(1)Universidade Estadual Paulista, Faculdade de Engenharia, Departamento de Fitossanidade, Engenharia Rural e Solos, Avenida Brasil no 56, Caixa postal 31, CEP 15385-000 Ilha Solteira, SP. E-mail: renan_agro@yahoo.com.br, marineid@bio.feis.unesp.br, eloizaaziole@hotmail.com, max_olivm@hotmail.com
\end{abstract}

\begin{abstract}
Resumo - O objetivo deste trabalho foi identificar ácaros predadores em plantas de lichia e correlacionar o desenvolvimento populacional dessas espécies com o do ácaro-da-erinose da lichia, Aceria litchii. A pesquisa foi desenvolvida no Município de Casa Branca, SP, com árvores adultas, de 12 anos de idade, da variedade Bengal. Mensalmente, de agosto de 2008 a setembro de 2009, foram coletadas folhas para avaliação dos níveis populacionais de $A$. litchii e de ácaros predadores. Foram registrados 6.557 indivíduos da família Phytoseiidae. A espécie mais abundante foi Amblyseius compositus (42,6\%), seguida por Phytoseius intermedius (31,2\%), Euseius concordis $(14,1 \%)$, Amblyseius herbicolus $(8,8 \%)$ e Iphiseiodes zuluagai $(3,3 \%)$. O desenvolvimento populacional de A. compositus, E. concordis e I. zuluagai correlacionou-se positivamente com o de Aceria litchii, o que indica relação de predação.
\end{abstract}

Termos para indexação: Aceria litchii, Litchi chinensis, controle biológico, desenvolvimento populacional, Eriophyidae, Phytoseiidae.

\section{Predator mites associated with the litchi erinose mite}

\begin{abstract}
The objective of this work was to identify predator mites present in lychee plants and to correlate these species population development with the one of the litchi erineum mite, Aceria litchii. The research was carried out in the county of Casa Branca, SP, Brazil, with mature trees of the Bengal variety, with 12 years old. From August 2008 to September 2009, leaves were collected monthly for the evaluation of $A$. litchii and predatory mites population levels. Registers were made for 6,557 mites of the Phytoseiidae family. The most abundant species was Amblyseius compositus (42.6\%), followed by Phytoseius intermedius (31.2\%), Euseius concordis (14.1\%), Amblyseius herbicolus (8.8\%) and Iphiseiodes zuluagai (3.3\%). Population development of A. compositus, E. concordis and I. zuluagai was positively correlated with the one of Aceria litchii, indicating a predation relationship.
\end{abstract}

Index terms: Aceria litchii, Litchi chinensis, biological control, population development, Eriophyidae, Phytoseiidae.

\section{Introdução}

No Brasil, o cultivo da lichia, Litchi chinensis Sonn (Sapindaceae), é recente e concentrado no Estado de São Paulo. Na safra 2007/2008, com exceção da cultura da laranja, o estado paulista contava com 191.726,24 hectares cultivados com espécies frutíferas (São Paulo, 2008), dos quais 1.615,30 hectares com a cultura da lichia, uma área superior à ocupada por culturas como a de acerola (597,4 ha), mamão (583,8 ha) e pinha (883,0 ha). Em razão de seu alto valor econômico (Centrais de Abastecimento de Campinas, 2009), a viabilização dessa cultura pode representar um importante acréscimo de renda para o produtor.

$\mathrm{Na}$ China, Índia, Tailândia e Vietnã, o ácaro-da-erinose, Aceria litchii (Keifer), é o maior problema encontrado na produção de lichia (Siddiqui, 2002). O seu ataque em folhas jovens causa a produção de eríneos - tricomas desenvolvidos anormalmente-, na superfície abaxial das folhas, que posteriormente se transformam em galhas. Essas galhas são como pequenas bolhas, que podem crescer, cobrir a folha por inteiro e causar encrespamento. Em muitos casos, todo limbo foliar pode ser deformado (Food and Agriculture Organization, 2002). A infestação de A. litchii pode comprometer severamente a produção de lichia. Portanto, a viabilização dessa cultura passa pelo desenvolvimento de estratégias de manejo desse ácaro.

Pouco se conhece sobre os padrões de ocorrência sazonal e de diversidade de comunidades de ácaros associados a plantas de lichia no Brasil, conhecimento esse que é básico e imprescindível na elaboração de 
propostas de manejo de espécies que se tornaram praga. O primeiro levantamento de ácaros em plantas de lichia realizado no Estado de São Paulo não constatou a presença do ácaro-da-erinose (Mineiro \& Raga, 2003). Contudo, em julho e agosto de 2007, Raga et al. (2010) o detectaram pela primeira vez no Brasil, nos municípios de Casa Branca e Tambaú, no Estado de São Paulo.

No levantamento realizado por Mineiro \& Raga (2003), nos municípios de Sumaré, Regente Feijó, Campinas, Atibaia e Tietê, foi registrada, pela primeira vez em plantas de lichia no Brasil, a ocorrência de 19 espécies de ácaros pertencentes a 9 famílias: Phytoseiidae, Stigmaeidae, Bdellidae, Eupalopsellidae, Anystidae, Tetranychidae, Tuckerellidae, Tenuipalpidae e Tydeidae. A família Phytoseiidae apresentou a maior diversidade, com cinco espécies: Amblyseius herbicolus (Chant), Euseius citrifolius (Denmark \& Muma), Euseius concordis (Chant), Iphiseiodes zuluagai (Denmark \& Muma) e Phytoseiulus macropilis (Banks). Foram registradas duas espécies de Stigmaeidae, Agistemus sp. e Zetzellia sp.

Ácaros da família Phytoseiidae têm sido registrados em associação com ácaros fitófagos em muitas culturas e atuam como importantes agentes de controle biológico (Moraes, 2002; Gerson et al., 2003). Algumas espécies de fitoseídeos são relatadas na literatura associadas a eriofídeos, inclusive com Aceria litchii. Os ácaros fitoseídeos Amblyseius herbicolus e A. eharai (Amitai \& Swirski) são considerados predadores do ácaro-da-erinose na Austrália e na China (Waite \& Gerson, 1994). Na Índia, Sharma \& Thakur (1992) registraram a ocorrência de Amblyseius largoensis (Muma), A. paraaerialis (Muma), Phytoseius intermedius (Evans \& Macfarlane), Typhlodromus homalii (Gupta) e T. fleschneri (Chant) em plantas infestadas com Aceria litchii.

O presente trabalho teve por objetivo identificar ácaros predadores em plantas de lichia e correlacionar seu desenvolvimento populacional ao de Aceria litchii.

\section{Material e Métodos}

A pesquisa foi desenvolvida no Município de Casa Branca, SP, em propriedade particular, a $21^{\circ} 47^{\prime} 12^{\prime \prime S}$ e $47^{\circ} 06^{\prime} 65^{\prime \prime} \mathrm{W}$ e $702 \mathrm{~m}$ de altitude. O clima do local de acordo com a classificação de Köppen é do tipo Aw - tropical com estação seca no inverno-, com temperatura média anual de $21,5^{\circ} \mathrm{C}$ e precipitação média anual de $1.310 \mathrm{~mm}$ (Centro de Pesquisas Meteorológicas e Climáticas Aplicadas, 2008).

A área experimental consistiu de quatro árvores de lichia adultas, de 12 anos de idade, da variedade Bengal, em um pomar com 110 lichieiras. De agosto de 2008 a setembro de 2009, foram coletados mensalmente de cada planta, no terço médio da copa, quatro extremidades de ramos com aproximadamente 0,50 m de comprimento, uma de cada quadrante, para avaliação de $A$. litchii, e duas extremidades de ramos de 0,30 m de comprimento, de quadrantes diferentes, para avaliação de ácaros predadores. O material coletado foi acondicionado em sacos de plástico, colocado em caixas de poliestireno e conduzido ao laboratório no mesmo dia. A avaliação foi realizada no prazo máximo de 36 horas após a coleta.

Nas avaliações de $A$. litchii, foram encontrados ácaros brancos e vermelhos. Lâminas com exemplares das duas cores foram encaminhadas, para identificação, à Embrapa Recursos Genéticos e Biotecnologia, Núcleo Temático de Segurança Biológica, em Brasília, DF. A contagem dessas duas formas foi realizada separadamente.

Os eriofídeos foram contados em gemas apicais e em galhas foliares. No caso das galhas, foram utilizadas duas folhas novas e duas maduras por ramo, e de cada uma delas foi cortada uma área de $2 \mathrm{~cm}^{2}$, com galhas. Esses pedaços foram colocados, individualmente, em solução de água e detergente neutro, a 0,5\%, e agitados por $5 \mathrm{~s}$, para desalojar os ácaros dos eríneos presentes nas galhas. Posteriormente, o número de ácaros brancos e vermelhos foi contado sob microscópio estereoscópico.

Para a contagem do número de ácaros presentes nas gemas, foram utilizadas as gemas apicais dos ramos coletados, individualizadas em recipiente com solução de água com detergente neutro a $0,5 \%$, agitadas por alguns segundos para posterior contagem em estereoscópio.

No caso dos predadores, as extremidades de ramos, separadas por planta, foram colocadas inteiras em recipiente com solução de água e detergente a $0,5 \%$, conforme metodologia adaptada de Návia (2010). Após agitação durante $15 \mathrm{~s}$, a amostra permaneceu em repouso por $5 \mathrm{~min}$. A solução de detergente e as partículas desprendidas e sedimentadas foram vertidas 
sobre um jogo de peneiras granulométricas, com $21 \mathrm{~cm}$ de diâmetro e malha/abertura de: 16 Mesh/1 mm, 32 $\mathrm{Mesh} / 500 \mu \mathrm{m}, 270 \mathrm{Mesh} / 53 \mu \mathrm{m}$. A peneira superior foi lavada com água abundante e em alta pressão, para facilitar a passagem das partículas menores que a abertura da peneira. As partículas retidas nas duas peneiras de menor abertura, onde estavam os ácaros, foram transferidas para um frasco com solução de álcool etílico a 70\%, com o uso de um jato de álcool a $70 \%$ de uma pisseta.

A solução resultante do processo de lavagem, com as partículas retidas das duas peneiras, foi examinada ao microscópio estereoscópico, e os ácaros encontrados foram montados em lâminas de microscopia, com meio de Hoyer (Moraes \& Flechtmann, 2008), para posterior identificação e contagem. Lâminas de cada tipo de ácaro da família Phytoseiidae foram encaminhadas ao Departamento de Zoologia e Botânica da Unesp, Campus de São José do Rio Preto, para identificação.

Os dados climáticos de temperatura, evapotranspiração e pluviosidade foram fornecidos pelo Centro de Pesquisas Meteorológicas e Climáticas Aplicadas (2008) que, nesse município, possui um ponto de coleta automatizado.

Os dados de número de ácaros predadores, por planta e por espécie, registrados ao longo do período de avaliação, foram transformados em $\log (x+2)$, e as médias mensais foram agrupadas pelo teste de Scott-Knott, a 5\% de probabilidade.

Para cada espécie de ácaro predador, o número de espécimes coletados por planta foi correlacionado aos números de $A$. litchii de coloração branca e vermelha presentes em galhas de folhas novas e maduras, e em gemas. Os dados de cada espécie foram correlacionados aos dados climáticos. Todas as correlações foram feitas com o uso do índice de correlação linear de Pearson (Gomes, 2009), a 5\% de probabilidade.

As análises estatísticas foram realizadas com o uso do programa Sisvar (Ferreira, 2008).

\section{Resultados e Discussão}

Os exemplares de Eriophyidae, de coloração branca e vermelha, foram identificados como Aceria litchii (Keifer). Adultos com cor e tamanhos diferentes já foram observados em eriofídeos, como em Aceria tulipae (Keifer), de acordo com o registro de Somsen (1966), que relatou a presença de uma forma migratória, provavelmente associada a mudanças na temperatura e na qualidade ou quantidade do alimento, que poderia ser diferenciada da forma principal, com função reprodutiva, pelo tamanho, cor e por se movimentar mais ativamente sobre a planta. Assim, a diferença de coloração observada em $A$. litchii talvez seja um indício da existência de uma fase reprodutiva e de uma fase adaptada à dispersão. Entretanto, maiores observações são necessárias para explicar essa diferença no ácaro-da-erinose da lichia.

Foram registrados 6.557 fitoseídeos de setembro de 2008 a agosto de 2009. As espécies de Phytoseiidae encontradas foram: Amblyseius compositus (Denmark \& Muma), A. herbicolus, Euseius concordis, Iphiseiodes zuluagai e Phytoseiulus macropilis. Além dos fitoseídeos, foram registradas mais três espécies, Brevipalpus phoenicis (Geijskes), Oligonychus sp. e uma espécie de Iolinidae, mas todas em pequena quantidade, no total de 16, 39 e 13 indivíduos, respectivamente.

As espécies mais abundantes foram A. compositus, com $42,6 \%$ do total de fitoseídeos coletados, e P. intermedius com 31,2\%. Euseius concordis teve $14,1 \%$, A. herbicolus $8,8 \%$ e I. zuluagai 3,3\%. Dessas espécies, as três últimas já tinham sido registradas em plantas de lichia, no Estado de São Paulo, por Mineiro \& Raga (2003). Phytoseiulus intermedius foi relatado apenas nas regiões produtoras da China e Índia (Sharma \& Thakur, 1992) e, pela primeira vez, o ácaro A. compositus foi observado nessa frutífera.

O número de ácaros predadores coletados variou ao longo do ano (Tabela 1). Phytoseiulus intermedius apresentou a maior ocorrência em outubro e dezembro de 2008, época de maior precipitação, e $A$. herbicolus em julho de 2009, quando a temperatura diminuiu (Figura 1 A). Essas duas espécies foram correlacionadas a fatores climáticos, a primeira foi favorecida pela pluviosidade, e a segunda foi prejudicada pelo aumento da temperatura e da evapotranspiração (Tabela 2). O aumento da temperatura correlacionouse negativamente ao número de formas brancas de $A$. litchii, em folhas novas de lichia. As outras espécies não tiveram correlação com os fatores climáticos.

As populações dos predadores A. compositus, E. concordis e o I. zuluagai apresentaram correlações altas e positivas com o ácaro-da-erinose da lichia (Tabela 3). Essas três espécies atingiram o pico populacional em outubro de 2008 (Figura 1 B), que 
coincidiu com os picos populacionais das formas brancas e vermelhas de $A$. litchii. O período de maior ocorrência de $A$. litchii coincidiu com a fase de frutificação da lichieira (Tabela 1), e a população de formas vermelhas, em outubro de 2008, foi três vezes superior à de formas brancas.

A correlação entre as populações de $A$. compositus, E. concordis e I. zuluagai e a população de $A$. litchii é indicação de que essas espécies podem utilizar esse eriofídeo como fonte alimentar, principalmente
A. compositus, que totalizou $42,6 \%$ dos fitoseídeos coletados. No Brasil, esse ácaro tem sido registrado em culturas como cafeeiro (Mineiro et al., 2009), mamoeiro (Vasconcelos et al., 2006) e seringueira (De Vis et al., 2006). Em cafeeiro, tem sido uma espécie frequente e capaz de predar o ácaro da mancha-anular Brevipalpus phoenicis (Geijskes) (Reis et al., 2007). Euseius concordis e I. zuluagai são espécies comumente encontradas em citros, consideradas como importantes predadores dos ácaros-praga da cultura (Gravena,

Tabela 1. Número médio de ácaros da família Phytoseiidae, por planta, e estádios fenológicos de planta de lichia, de setembro de 2008 a agosto de $2009^{(1)}$.

\begin{tabular}{|c|c|c|c|c|c|c|c|}
\hline \multirow[t]{2}{*}{ Datas } & \multicolumn{6}{|c|}{ Número médio de ácaros ${ }^{(2)}$} & \multirow[t]{2}{*}{ Estádios fenológicos } \\
\hline & Amblyseius compositus & Amblyseius herbicolus & Euseius concordis & Iphiseiodes zuluagai & Phytoseiulus intermedius & Total & \\
\hline Setembro & $131 b$ & $2 \mathrm{f}$ & $2 d$ & $2 \mathrm{c}$ & $62 b$ & $198 b$ & Frut \\
\hline Outubro & $335 \mathrm{a}$ & $24 b$ & $68 \mathrm{a}$ & $24 \mathrm{a}$ & $91 \mathrm{a}$ & $543 \mathrm{a}$ & Frut \\
\hline Novembro & $27 \mathrm{c}$ & $5 \mathrm{e}$ & $16 b$ & $2 \mathrm{c}$ & $16 \mathrm{c}$ & $65 c$ & Frut \\
\hline Dezembro & $95 b$ & $2 \mathrm{f}$ & $25 b$ & $2 \mathrm{c}$ & $257 \mathrm{a}$ & $381 b$ & $\mathrm{RP}$ \\
\hline Janeiro & $10 \mathrm{~d}$ & $2 \mathrm{f}$ & $33 a$ & $8 b$ & $19 \mathrm{c}$ & $71 \mathrm{c}$ & $\mathrm{RP}$ \\
\hline Feveiro & $24 \mathrm{c}$ & $2 f$ & $24 \mathrm{~b}$ & $2 \mathrm{c}$ & $2 \mathrm{e}$ & $55 \mathrm{c}$ & EFV \\
\hline Março & $11 d$ & $11 \mathrm{c}$ & $35 \mathrm{a}$ & $11 b$ & $35 b$ & $102 \mathrm{c}$ & EFV \\
\hline Abril & $22 \mathrm{c}$ & $11 \mathrm{c}$ & $13 b$ & $8 b$ & $8 \mathrm{~d}$ & $61 \mathrm{c}$ & EFV \\
\hline Maio & $22 c$ & $7 \mathrm{~d}$ & $7 \mathrm{c}$ & $2 c$ & $12 \mathrm{c}$ & $50 \mathrm{c}$ & MB \\
\hline Junho & $4 \mathrm{e}$ & $23 b$ & $9 \mathrm{c}$ & $2 \mathrm{c}$ & $8 d$ & $45 c$ & EFR \\
\hline Julho & $22 c$ & $53 a$ & $17 \mathrm{~b}$ & $7 b$ & $7 d$ & $107 \mathrm{c}$ & EFR \\
\hline Agosto & $19 \mathrm{c}$ & $29 b$ & $7 \mathrm{c}$ & $9 b$ & $19 \mathrm{c}$ & $83 c$ & Flor \\
\hline CV (\%) & 16,4 & 12,9 & 18,1 & 15,1 & 18,4 & 13,4 & \\
\hline
\end{tabular}

${ }^{(1)}$ Médias seguidas de letras iguais na coluna não diferem pelo teste Scott-Knott, a $5 \%$ de probabilidade. ${ }^{(2)}$ Médias originais; dados transformados em log (x+2) para análise estatísitca. Frut, frutificação; RP, repouso da planta; EFV, emissão de fluxo vegetativo; MB, maturação dos brotos; EFR, emissão de fluxo reprodutivo; Flor, florescimento.

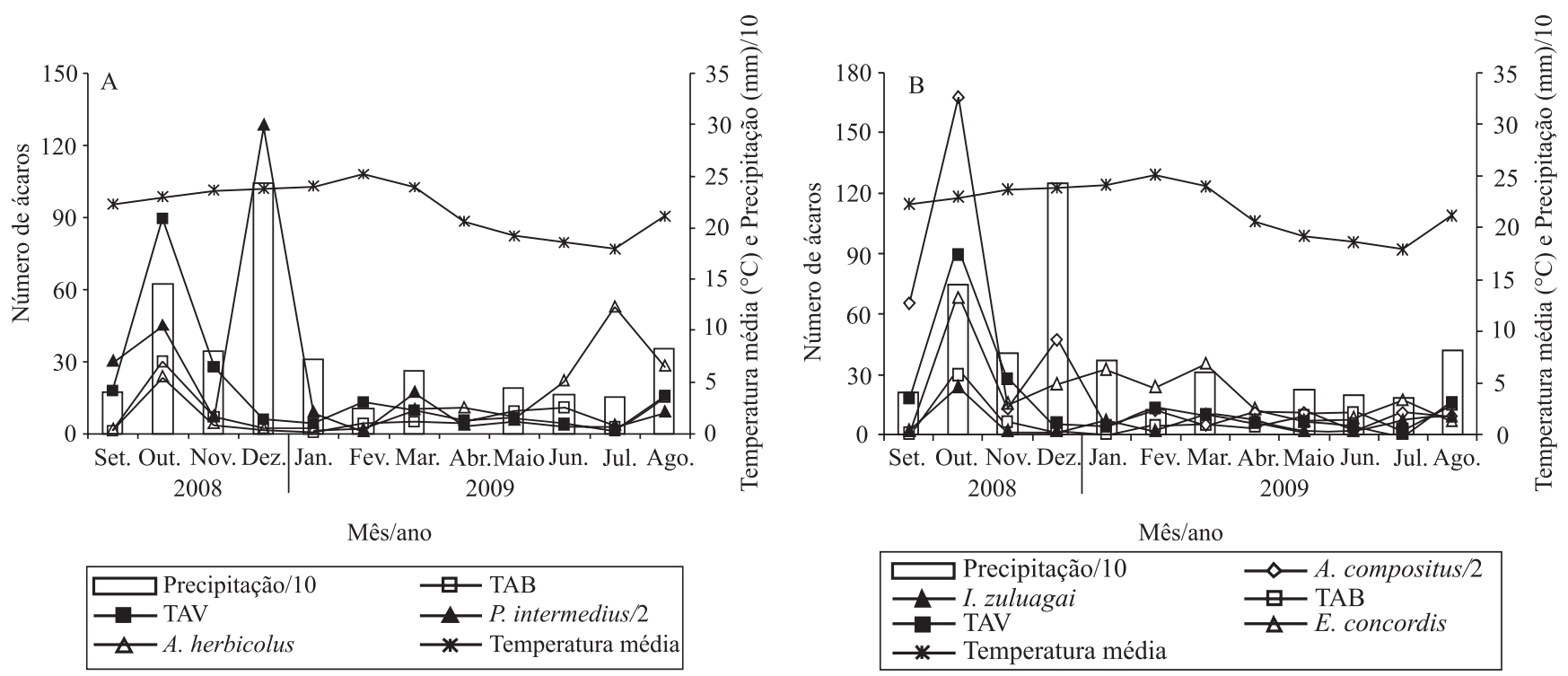

Figura 1. Número total de formas brancas (TAB) e vermelhas (TAV) de Aceria litchii por planta, número médio de Amblyseius herbicolus e Phytoseiulus intermedius (A) e de Amblyseius compositus, Euseius concordis e Iphiseiodes zuluagai (B) por planta, temperatura máxima média e precipitação total em Casa Branca, SP. 
2005). Essas espécies apresentaram correlação positiva com a população do eriofídeo Dichopelmus notus (Keifer), em um levantamento populacional realizado em erva-mate, no Estado do Paraná (Gouvea et al., 2006).

Essas três espécies de fitoseídeos são reconhecidas como ácaros predadores generalistas. De acordo com McMurtry \& Croft (1997), A. compositus e $P$. intermedius são classificados como predadores do tipo III, porque podem se alimentar de pequenos insetos, ácaros de diferentes famílias, inclusive eriofídeos, e também de pólen e exsudatos vegetais, enquanto que $E$. concordis é um predador do tipo IV, por ter preferência por grãos de pólen, embora também possa ingerir diferentes tipos de presas. Em lichia, as plantas floresceram em agosto; em setembro, teve início a infestação de $A$. litchii e as populações de fitoseídeos começaram a crescer. Aceria litchii e os predadores atingiram os maiores níveis em outubro de 2008. É possível que parte do crescimento populacional de Amblyseius compositus, E. concordis e I. zuluagai tenha sido influenciado pela presença de pólen no mês de agosto, mas os índices de correlação entre essas espécies e $A$. litchii indicam a ocorrência de uma relação de predação.

Para algumas espécies da família Phytoseiidae, ácaros eriofídeos podem representar uma alimentação favorável, enquanto para outras podem não ser adequados (McMurtry \& Rodriguez, 1987). Segundo Moraes \& Lima (1983), a alimentação de E. concordis com Aculops lycopersici (Massee) pode resultar em grande sucesso reprodutivo. Na Austrália e na China, Amblyseius herbicolus e A. eharai são considerados predadores do ácaro-da-erinose Aceria litchii (Waite \& Gerson, 1994).

A formação de eríneos, como ocorre no caso do ataque de A. litchii em lichieira, pode representar uma proteção contra a predação (Sabelis, 1996). Entretanto, esses locais são frequentados por ácaros predadores, possivelmente porque têm sucesso na captura de presas (Waite \& Gerson, 1994). Apesar dessa proteção, em algum momento do seu ciclo biológico, os eriofídeos têm de sair desse refúgio para colonizar outros locais e, durante essa migração, ficam expostos a condições climáticas adversas e aos predadores. Além disso, a formação das novas galhas pode levar

Tabela 2. Coeficiente de correlação entre dados climáticos e os números de ácaros de Aceria litchii de coloração branca, encontrados em folhas novas, e de ácaros fitoseídeos presentes em ramos de plantas de lichia.

\begin{tabular}{|c|c|c|c|c|c|c|c|c|c|}
\hline \multirow[t]{2}{*}{ Ácaros } & \multicolumn{9}{|c|}{ Dados climáticos $^{(1)}$} \\
\hline & TA & $\mathrm{Ta}$ & TM & $\mathrm{Tm}$ & $\mathrm{T}$ & ETP & $\mathrm{P}$ & PA & NDC \\
\hline Aceria litchii (brancos) & $-0,564$ & $-0,462$ & $-0,629 *$ & $-0,444$ & $-0,539$ & $-0,585^{*}$ & $-0,326$ & $-0,195$ & $-0,251$ \\
\hline Amblyseius compositus & 0,419 & 0,121 & 0,280 & 0,151 & 0,212 & 0,262 & 0,345 & 0,176 & $-0,009$ \\
\hline Amblyseius herbicolus & $-0,543$ & $-0,665^{*}$ & $-0,604 *$ & $-0,696^{*}$ & $-0,675^{*}$ & $-0,636^{*}$ & $-0,289$ & $-0,239$ & $-0,351$ \\
\hline Euseius concordis & 0,422 & 0,489 & 0,410 & 0,482 & 0,463 & 0,515 & 0,293 & 0,147 & 0,183 \\
\hline Iphiseiodes zuluagai & 0,207 & 0,101 & 0,128 & 0,083 & 0,104 & 0,063 & $-0,012$ & $-0,108$ & $-0,226$ \\
\hline Phytoseiulus intermedius & 0,318 & 0,318 & 0,289 & 0,340 & 0,327 & 0,378 & $0,937 * *$ & $0,882 * *$ & 0,573 \\
\hline
\end{tabular}

${ }^{(1)} \mathrm{TA}$, temperatura máxima absoluta; Ta, temperatura mínima absoluta; TM, temperatura média máxima; Tm, temperatura média mínima; T, temperatura média; ETP, evapotranspiração potencial; P, precipitação mensal; PA, precipitação mensal acumulada; NDC, número de dias com chuva no mês. * e ** Significativo a 5 e $1 \%$ de probabilidade, pelo teste de correlação linear de Pearson, respectivamente.

Tabela 3. Coeficiente de correlação entre número de ácaros de Aceria litchii, encontrados em gemas e galhas foliares de folhas novas e maduras, e de ácaros fitoseídeos presentes em ramos de planta de lichia.

\begin{tabular}{|c|c|c|c|c|c|c|c|c|}
\hline \multirow[t]{2}{*}{ Ácaros } & \multicolumn{8}{|c|}{ Formas encontradas de Aceria litchii $^{(1)}$} \\
\hline & TAB & TAV & $\mathrm{BN}$ & $\mathrm{BM}$ & $\mathrm{BGa}$ & VGa & $\mathrm{BGe}$ & VGe \\
\hline Amblyseius compositus & $0,685^{*}$ & $0,900 * *$ & $-0,022$ & $0,851 * *$ & $0,679 *$ & $0,867 * *$ & $0,598 *$ & 0,465 \\
\hline Amblyseius herbicolus & 0,331 & 0,087 & 0,329 & 0,239 & 0,326 & 0,083 & 0,305 & 0,228 \\
\hline Euseius concordis & 0,559 & $0,730 * *$ & $-0,173$ & $0,746^{* *}$ & 0,533 & $0,783 * *$ & $0,677^{*}$ & 0,464 \\
\hline Iphiseiodes zuluagai & $0,760 * *$ & $0,778 * *$ & 0,096 & $0,865 * *$ & $0,738 * *$ & $0,776 * *$ & $0,806^{* *}$ & $0,670 *$ \\
\hline Phytoseiulus intermedius & 0,012 & 0,161 & $-0,321$ & 0,167 & 0,005 & 0,177 & 0,076 & $-0,002$ \\
\hline
\end{tabular}

${ }^{(1)} \mathrm{TAB}$, total de formas brancas; TAV, total de formas vermelhas; BN, formas brancas em folhas novas; BM, formas brancas em folhas maduras; BGa, formas brancas em galhas; VGa, formas vermelhas em galhas; BGe, formas brancas em gemas; VGe, formas vermelhas em gemas. * e ** Significativo a 5 e $1 \%$ de probabilidade, pelo teste de correlação linear de Pearson, respectivamente. 
de algumas horas a alguns dias e, nesse período, os ácaros estão vulneráveis (Sabelis, 1996). No entanto, são necessários mais estudos para determinar se essa predação pode ter papel relevante no controle dessa praga.

Phytoseius intermedius, espécie abundante nas plantas de lichia, neste estudo não apresentou correlação com a população de $A$. litchii, mas foi correlacionada positivamente à precipitação. O gênero Phytoseius tem sido associado à presença de tricomas nas folhas, como no levantamento realizado por Walter (1992) em florestas da Austrália, em que, de 720 espécimes coletados desse gênero, 714 estavam associados a plantas com folhas pubescentes. Em comparação aos outros fitoseídeos, os ácaros desse gênero são pequenos, possuem corpo mais estreito, pernas longas, características que podem ajudá-los a penetrar em áreas com tricomas. Assim, apesar de não ter tido correlação com o número de espécimes de $A$. litchii, $P$. intermedius pode ter sido favorecido pelo desenvolvimento dos eríneos pela infestação do eriofídeo. É possível, inclusive, que tenha ocorrido alguma predação, uma vez que outras espécies desse gênero já foram observadas predando eriofídeos. Em videiras na Itália, Phytoseius finitimus Ribaga e P. plumifer (C. \& F.) podem se alimentar de Colomerus vitis (Pagenstecher) (Duso \& De Lillo, 1996).

A correlação encontrada entre as populações de A. litchii e as dos ácaros predadores Amblyseius compositus, E. concordis e I. zuluagai evidencia a importância desses ácaros predadores, o que deve estimular mais estudos que confirmem a eficiência dessa predação. Estratégias que possam favorecer a manutenção dessas espécies nas áreas cultivadas devem ser desenvolvidas. Por serem ácaros generalistas, que podem se alimentar de pólen, uma possibilidade a ser testada é a do uso de espécies produtoras de pólen como quebra-ventos.

\section{Conclusões}

1. O aumento populacional dos ácaros predadores Amblyseius compositus, Euseius concordis e Iphiseiodes zuluagai se correlaciona positivamente ao aumento no número de Aceria litchii.

2. Os ácaros A. compositus, E. concordis eI. zuluagai são predadores promissores para programas de manejo do ácaro-da-erinose.

\section{Agradecimentos}

À Dra. Denise Návia, da Embrapa Recursos Genéticos e Biotecnologia, Núcleo Temático de Segurança Biológica, pela identificação de A. litchii; e ao Dr. Antonio Carlos Lofego, da Universidade Estadual Paulista, Campus de São José do Rio Preto, pela identificação dos ácaros da família Phytoseiidae.

\section{Referências}

CENTRAIS DE ABASTECIMENTO DE CAMPINAS. Informações técnico-econômicas: boletim informativo diário de preços. Campinas: CEASA, 2009. 4p. (Ceasa. Boletim, 551).

CENTRO DE PESQUISAS METEOROLÓGICAS E CLIMÁTICAS APLICADAS. Clima dos municípios paulistas: Casa Branca. Disponível em: <http://www.cpa.unicamp.br/outras-informacoes/ clima_muni_124.html >.Acesso em: 20 set. 2008.

DE VIS, R.M.J.; MORAES, G.J. de; BELLINI, M.R. Mites (Acari) of rubber trees (Hevea brasiliensis Muell. Arg., Euphorbiaceae) in Piracicaba, State of São Paulo, Brazil. Neotropical Entomology, v.35, p.112-120, 2006.

DUSO, C.; DE LILLO, E. Grape. In: LINDQUIST, E.E.; SABELIS, M.W.; BRUIN, J. Eriophyoid mites: their biology, natural enemies, and control. Amsterdam: Elsevier, 1996. p.571-582. (World crop pests, 6).

FERREIRA, D.F. SISVAR: um programa para análises e ensino de estatística. Revista Symposium, v.6, p.36-41, 2008.

FOOD AND AGRICULTURE ORGANIZATION. Expert consultation on lychee production in the Asia-Pacific Region. Rome: FAO, 2002. 88p.

GERSON, U.; SMILEY, R.L.; OCHOA, R. Mites (Acari) for pest control. Oxford: Blackwell Sciences, 2003. 539p.

GOMES, F.P. Curso de estatística experimental. 15.ed. Piracicaba: FEALQ, 2009. 451p.

GOUVEA, A. de; BOARETTO, L.C.; ZANELLA, C.F.; ALVES, L.F.A. Dinâmica populacional de ácaros (Acari) em erva-mate(Ilexparaguariensis St. Hil.:Aquifoliaceae). Neotropical Entomology, v.35, p.101-111, 2006.

GRAVENA, S. Manual prático de manejo ecológico de pragas dos citros. Jaboticabal: Gravena, 2005. 372p.

LOFEGO, A.C.; MORAES, G.J. de. Ácaros (Acari) associados a mirtáceas (Myrtaceae) em áreas de cerrado no Estado de São Paulo com análise faunística das famílias Phytoseiidae e Tarsonemidae. Neotropical Entomology, v.35, p.731-746, 2006.

MCMURTRY, J.A.; CROFT, B.A. Life-styles of phytoseiid mites and their roles in biological control. Annual Review of Entomology, v.42, p.291-321, 1997.

MCMURTRY, J.A.; RODRIGUEZ, J.G. Nutritional ecology of phytoseiid mites. In: SLANSKY JUNIOR, F.; RODRIGUEZ, J.G. (Ed.). Nutritional ecology of insects, mites, spiders and related invertebrates. New York: Wiley \& Sons, 1987. p.609-644. 
MINEIRO, J.L. de C.; RAGA, A. Ocorrência de ácaros (Arachnida: Acari) em plantas de lichia (Litchi chinensis Sonn.) (Sapindaceae) no Estado de São Paulo. In: REUNIÃO ANUAL DO INSTITUTO BIOLÓGICO, 16.; CONGRESSO DE INICIAÇÃO CIENTÍFICA EM CIÊNCIAS AGRÁRIAS, BIOLÓGICAS E AMBIENTAIS, 1., 2003, São Paulo. Anais. São Paulo: Instituto Biológico, 2003. p.1-4.

MINEIRO, J.L. de C.; RAGA, A.; SATO, M.E.; LOFEGO, A.C. Ácaros associados ao cafeeiro (Coffea spp.) no Estado de São Paulo, Brasil. Parte I. Mesostigmata. Biota Neotropical, v.9 p.37-46, 2009.

MORAES, G.J. Controle biológico de ácaros fitófagos com ácaros predadores. In: PARRA, J.R.P.; BOTELHO, P.S.M.; CORRÊA-FERREIRA, B.S.; BENTO, J.M.S. (Ed.). Controle biológico no Brasil: parasitóides e predadores. São Paulo: Manole, 2002. p.225-237.

MORAES, G.J. de; FLECHTMANN, C.H.W. Manual de acarologia, acarologia básica e ácaros de plantas cultivadas no Brasil. Ribeirão Preto: Holos, 2008. 288p.

MORAES, G.J. de; LIMA, H.C. Biology of Euseius concordis (Chant), a predator of the tomato russet mite. Acarologia, v.24, p.251-255, 1983.

NÁVIA, D. Procedimentos: método de lavagem. Brasília: Embrapa Recursos Genéticos e Biotecnologia, 2010.

RAGA, A.; MINEIRO, J.L. de C.; SATO, M.E.; MORAES, G.J. de; FLECHTMANN, C.H.W. Primeiro relato de Aceria litchii (Keifer) (Prostigmata: Eriophyidae) em plantas de lichia no Brasil. Revista Brasileira de Fruticultura, v.32, p.628-629, 2010.

REIS, P.R.; TEODORO, A.V.; PEDRO NETO, M. História de vida de Amblyseius compositus Denmark \& Muma predando Brevipalpus phoenicis (Geijskes) (Acari: Phytoseiidae, Tenuipalpidae). Coffee Science, v.2, p.150-158, 2007.
SABELIS, M.W. Phytoseiidae. In: LINDQUIST, E.E.; SABELIS, M.W.; BRUIN, J. Eriophyoid mites: their biology, natural enemies, and control. Amsterdam: Elsevier, 1996. p.427-456. (World crop pests, 6).

SÃO PAULO (Estado). Secretaria de Agricultura e Abastecimento. Coordenadoria de Assistência Técnica Integral. Instituto de Economia Agrícola. Levantamento censitário de unidades de produção agrícola do Estado de São Paulo - LUPA 2007/2008. São Paulo: SAA/CATI/IEA, 2008. Disponível em: <http://www. cati.sp.gov.br/projetolupa>. Acesso em: 17 maio 2010.

SHARMA, D.D.; THAKUR, A.P. Bioefficacy of eight pesticides against litchi erineum mite (Aceria litchii) and its predators. Indian Journal of Agricultural Sciences, v.62, p.240-242, 1992.

SIDDIQUI, S.B.M.A.B. Lychee production in Bangladesh. In: PAPADEMETRIOU, M.K.; DENT, F.J. Lychee production in the Asia-Pacific Region. Bangkok: FAO, 2002. p.28-40.

SOMSEN, H.W. Development of migratory form of wheat curl mite. Journal of Economic Entomology, v.59, p.1283-1284, 1966.

VASCONCELOS, G.J.N.; SILVA, F.R.; BARBOSA. D.G.F.; GONDIN JUNIOR, M.G.C.; MORAES, G.J. Diversidade de fitoseídeos (Acari: Phytoseiidae) em fruteiras tropicais no Estado de Pernambuco, Brasil. Magistra, v.18, p.90-101, 2006.

WAITE, G.K.; GERSON, U. The predator guild associated with Aceria litchii (Acari: eriophyidae) in Australia and China. Entomophaga, v.39, p.275-280, 1994.

WALTER, D.E. Leaf surface-structure and the distribution of Phytoseius mites (Acarina, Phytoseiidae) in South-Eastern Australian forests. Australian Journal of Zoology, v.40, p.593-603, 1992.

Recebido em 19 de julho de 2010 e aprovado em 18 de outubro de 2010 